\title{
INTELIGENCIAS MÚLTIPLES Y ESTILOS DE APRENDIZAJE, SU RELACIÓN CON EL RENDIMIENTO ACADÉMICO DE ESTUDIANTES EN ESTADÍSTICA
}

\section{MULTIPLE INTELLIGENCES AND LEARNING STYLES, THEIR RELATIONSHIP WITH ACADEMIC PERFORMANCE OF STUDENTS IN STATISTICS}

\author{
José Joaquín Martínez-Lozano $^{1} \mid$ Mawency Vergel-Ortega² $^{2}$ Henry de Jesús Gallardo-Pérez ${ }^{3}$
}

Forma de citar: MARTÍNEZ-LOZANO José, VERGEL-ORTEGA Mawency, GALLARDO-PÉREZ Henry. Inteligencias múltiples y estilos de aprendizaje, su relación con el rendimiento académico de estudiantes en estadística. Eco.Mat. 2014; 5(1): 74-86.

Recibido:

Junio 18 de 2014

Aceptado:

Agosto 15 de 2014

74

\section{RESUMEN}

La presente investigación se realizó con el propósito de determinar la relación entre inteligencias múltiples, estilos de aprendizaje al rendimiento académico de estudiantes de estadística de la Escuela Superior de Administración Pública seccional Cúcuta. Lo anterior en busca de implementar acciones que permitan mejorar el aprendizaje de la estadística por parte de los estudiantes, y, por supuesto las competencias en esta disciplina. Se realiza un estudio cuantitativo correlacional en una muestra de veinte tres estudiantes. Los resultados muestran asociaciones entre estilos de aprendizaje y su rendimiento, no obstante no existe relación significativa entre el tipo de inteligencia y el rendimiento en estos estudiantes. La metodología implementada permitió desarrollo de competencias al establecer roles acorde al tipo de inteligencia de los y las jóvenes estudiantes.

Palabras clave: Estadística, Inteligencias múltiples, competencias, Rendimiento académico.

\section{ABSTRACT}

This research was conducted in order to identify the factors associated with academic performance statistics students of the School of Public Administration Sectional Cúcuta. Also relate to the type of student intelligence and learning style. This looking to implement actions that will improve the learning of statistics by the students, and of course the skills in this discipline. Quantitative correlational study was conducted on a sample of twenty three students. The results show associations between learning styles and performance.

Escuela Superior de Administración Pública

${ }^{2}$ Universidad Francisco de Paula Santander

${ }^{3}$ Universidad Francisco de Paula Santander
Keywords: Statistical, Multiple Intelligences, competences, Academic achievement. 


\section{INTRODUCCIÓN}

$\mathrm{E}$

rendimiento académico de los estudiantes es una problemática que enfrentan los docentes en cursos con estudiantes de diferentes edades, género y ocupación. Cuando un docente asume un curso prepara un plan de clase para orientar a sus estudiantes con la misma metodología y las mismas estrategias pedagógicas, no obstante al llegar al aula e iniciar los procesos pedagógicos encuentra jóvenes con alto rendimiento y jóvenes con bajo rendimiento, algunos interpretan rápidamente resultados otros no, y algunos estudiantes no llegan a adquirir competencias básicas del programa.

Con respecto al caso mencionado, la presente investigación pretende determinar factores asociados, la relación existente entre el tipo de inteligencia de los estudiantes, su estilo de aprendizaje y los resultados de rendimiento académico obtenido en un curso de estadística en estudiantes de administración pública. Se procuró también determinar variables que inciden en el aprendizaje de la estadística según el modelo por situaciones problema implementado por el docente de la Escuela Superior de Administración Pública ESAP sede Cúcuta. ¿Qué factores están asociados al rendimiento académico de los estudiantes? Para ello se busca responder ¿Qué tipos de inteligencias múltiples poseen los estudiantes? ¿Cómo es el rendimiento académico de los estudiantes de Administración Pública en el curso de Estadística? ¿Qué efectos producen las inteligencias múltiples en el rendimiento académico en estadística en los estudiantes de Administración pública de la ESAP?

Cuando se refiere al estilo de aprendizaje de un estudiante, se hace alusión a rasgos cognitivos, afectivos y fisiológicos que sirven como indicador de cómo el estudiante percibe, interacciona y responde a ambientes de aprendizaje. Este estilo depende de tres parámetros, cómo el individuo selecciona la información, cómo la organiza y cómo trabaja la información. Según Krusche (1996), los canales sensoriales revisten importancia tanto en la percepción externa como en la percepción interna, dado que, así como se puede observar un objeto, también se puede imaginar el objeto, es decir, observarlo con los ojos internos.

Por otra parte, al conjunto de sentidos o sistema perceptivo el autor citado, lo denomina "VAKO", que no es otra cosa que la sigla compuesta por la primera letra de cada sentido, los cuales se utilizan para captar tanto el mundo exterior como el interior, estos mismos, se resumen de la siguiente manera. $\mathrm{V}=$ Visual $/$ ver; $\mathrm{A}=$ Auditivo / oir; $\mathrm{K}=$ Kinestésico / sensitivo; $\mathrm{O}=$ Olfativo y gustativo / oler y paladear. (p 67). Krusche (1996) afirma, "los seres humanos seleccionamos, entre las muchas informaciones que se nos ofrecen, aquellas que tienen trascendencia y significado para nosotros", quiere decir, que solo se registra una pequeña porción de todo lo que está alrededor y con lo cual se tiene contacto, además, la información seleccionada no es percibida con todos los sentidos, como también, sobre una misma situación no todas las personas se fijan en lo mismo, lo que hace la atención de las personas subjetiva o condicionada a gustos o intereses; pero de lo que se está seguro es de emplear preferentemente un canal sensorial específico o a lo sumo dos canales sensoriales; esto no es señal de que los demás canales no se utilicen, lo que sucede es que se hace de una forma menos consiente. ( $\mathrm{p} 68$ ).

Con referencia a lo planteado por Krusche (1996) el hecho de que en las escuelas la cantidad de estudiantes impide al maestro adaptarse a cada estudiante y a su forma de aprender, si podría con base en un estudio de caso mejorar el nivel académico y por supuesto de aprendizaje apelando a los diferentes sistemas perceptivos desde los más significativos hasta los menos significativos.
Enero-Diciembre 2014 ISSN 1794-8231

PP: $74-86$ 
No. 1

Enero-Diciembre 2014

ISSN 1794-8231

PP: $74-86$

En referencia a las inteligencias Múltiples, esta teorí $\neg$ a de Gardner (1983) favorece al máximo el desarrollo mental de los escolares y adolescentes. Se mencionan algunos lineamientos válidos a seguir en la identificación del perfil educacional del estudiante (valorar las fortalezas y debilidades a través de la observación). La identificación de talentos puede ser complementada con planes educacionales que busquen el total desenvolvimiento del educando.

Por otra parte, Gardner (1983) sostiene que existe un conjunto de competencias humanas relativamente autónomas que se denomina inteligencias humanas y que conforman la estructura intelectual del hombre. Estas inteligencias pueden ser modeladas $y$ combinadas de múltiples maneras. Es decir, amplía el campo de lo que es la inteligencia y reconoce lo que se sabía intuitivamente: que la brillantez académica no lo es todo. A la hora de desenvolverse en la vida no basta con tener un gran expediente académico. Hay gente de gran capacidad intelectual pero incapaz de, por ejemplo, elegir bien a sus amigos; por el contrario, hay gente menos brillante en el colegio que triunfa en el mundo de los negocios o en su vida personal. Triunfar en los negocios, o en los deportes, requiere ser inteligente, pero en cada campo se utiliza un tipo de inteligencia distinto. No mejor ni peor, pero sí distinto. En otras palabras, Einstein no es más ni menos inteligente que Pelé, simplemente sus inteligencias pertenecen a campos diferentes. Definir la inteligencia como una capacidad la convierte en una destreza que se puede desarrollar. No niega el componente genético, pero sostiene que esas potencialidades se van a desarrollar de una $\mathrm{u}$ otra manera dependiendo del medio ambiente, las experiencias vividas, la educación recibida, entre otras. Ningún deportista de élite llega a la cima sin entrenar, por buenas que sean sus cualidades naturales. Lo mismo se puede decir de los matemáticos, los poetas, o de la gente emocionalmente inteligente. Debido a eso, según el modelo propuesto por Howard Gardner todos los seres humanos están capacitados para el amplio desarrollo de su inteligencia, apoyados en sus capacidades y su motivación.

Howard Gardner y su equipo de la Universidad de Harvard han identificado ocho tipos distintos de inteligencia a saber: Inteligencia Lingüística, la que tienen los escritores, poetas, buenos redactores, utiliza ambos hemisferios. La inteligencia Lógico matemática, se utiliza para resolver problemas de lógica y matemáticas, es la inteligencia que tienen los científicos, se corresponde con el modo de pensamiento del hemisferio lógico y con lo que la cultura occidental ha considerado siempre como la única inteligencia. Inteligencia espacial, consiste en formar un modelo mental del mundo en tres dimensiones; es la inteligencia que tienen los marineros, los ingenieros, los cirujanos, los escultores, los arquitectos o los decoradores. Inteligencia musical, es aquella que permite desenvolverse adecuadamente a cantantes, compositores, músicos y bailarines.

De otra parte, la inteligencia corporal cinestésica, o capacidad de utilizar el propio cuerpo para realizar actividades o resolver problemas, es la inteligencia de deportistas, artesanos, cirujanos y bailarines. Inteligencia intrapersonal, es la que permite entenderse a sí mismo, no está asociada a ninguna actividad concreta. Inteligencia Interpersonal, permite entender a los demás; se suele encontrar en los buenos vendedores, políticos, profesores o terapeutas. Inteligencia naturalista, la utilizada cuando se observa y estudia la naturaleza, con el motivo de saber organizar, clasificar y ordenar, demostrada por biólogos o los herbolarios. Según esta teoría, todos los seres humanos poseen las ocho inteligencias en mayor o menor medida. Al igual que con los estilos de aprendizaje no hay tipos puros, y si los hubiera les resultaría imposible funcionar. 
Un ingeniero necesita una inteligencia espacial bien desarrollada, pero también necesita de todas las demás, de la inteligencia lógico matemática para poder realizar cálculos de estructuras, de la inteligencia interpersonal para poder presentar sus proyectos, de la inteligencia corporal - kinestésica para poder conducir un carro hasta la obra.

Todas las inteligencias son igualmente importantes y, según esto, el problema es que el sistema escolar vigente no las trata por igual sino que prioriza las dos primeras de la lista, (la inteligencia lógico - matemática y la inteligencia lingüística) hasta el punto de negar la existencia de las demás. Es evidente que, sabiendo lo que se sabe sobre estilos de aprendizaje, tipos de inteligencia y estilos de enseñanza, es absurdo que se siga insistiendo en que todos los alumnos aprendan de la misma manera. Contenidos similares se podrían presentar de formas muy diversas que permitan al alumno asimilarla partiendo de sus capacidades y aprovechando sus puntos fuertes.

Además, tendría que plantearse si una educación centrada en sólo dos tipos de inteligencia es la más adecuada para preparar a los alumnos para vivir en un mundo cada vez más complejo y con avances tecnológicos a una velocidad increíble.

\section{METODOLOGÍA}

Se realiza estudio enmarcado en el enfoque cuantitativo, descriptivo, de tipo campo, diseño correlacional. A 23 estudiantes que cursan asignatura de Estadística en la Escuela de Superior de Administración Pública ESAP. Se aplican pruebas de inteligencia múltiples, cuestionario de inteligencias múltiples de Howard Gardner test y el inventario de estilos de aprendizaje de Kolb, el cual permite al estudiante identificar características personales de la forma en cómo procesa la información.
En razón a la teoría anteriormente expuesta y con respecto a los estudiantes de estadística descriptiva en la Escuela Superior de Administración pública para el análisis de estilo de aprendizaje se plantearon como estudio de caso las preguntas, ¿cuáles son los modos de aprendizaje de estos estudiantes?, ¿cómo perciben más eficientemente la información?; para tal fin se aplicó un instrumento (test) de 15 preguntas, con base en las respuestas dadas por los estudiantes, se contó el número por cada código ( $\mathrm{a}, \mathrm{b}$, c); respuestas con a, corresponde a visual; respuestas con $\mathrm{b}$, corresponde a auditivo y respuestas con c corresponde a Kinestésico.

\section{RESULTADOS}

Los estudiantes ubicados en este nivel tienen bajas probabilidades de realizar lo descrito en el nivel básico de la asignatura. tienen mayor probabilidad de resolver acertadamente los ítems relacionados con la identificación de elementos básicos en lo referente a los contenidos estadística, determinación de medias, desviación, porcentajes, el dominio de la comprensión lectora es bajo o interpretación de resultados. En relación con el dominio de la expresión gráfica, por ejemplo, los estudiantes tienen altas posibilidades de graficar pero tampoco interpretan de manera correcta las mismas al enfrentarse a un objetivo de investigación.

Tras varios ensayos con los modelos cuyas variables explicaban mejor el rendimiento académico de los estudiantes, las variables independientes consideradas en este modelo, recabada la información mediante el cuestionario: edad, género, percepción de agrado o dificultad hacia la asignatura, hacinamiento, índice socioeconómico, expectativa de logro, motivación familiar, estado de la planta física e índice de servicios institucionales, recursos en el aula, dominio del tema del docente, índice de percepción de relaciones socioafectivas,
Enero-Diciembre 2014 ISSN $1794-8231$ PP: $74-86$ 
No. 1

Enero-Diciembre 2014

ISSN 1794-8231

PP: $74-86$

exigencia académica por parte del docente, tipo de inteligencia y estilo de aprendizaje. Variables relacionadas con el nivel mediante instrumento dirigido al docente, fueron consideradas: género, años de experiencia, grado académico, actualización, importancia brindada a la capacitación, tamaño de grupo, metodología, recursos para el desarrollo de la clase, frecuencia con que se asigna trabajos y tiempo destinado a la asignatura, así como actividades que se realizan en torno a ellas.

Existe evidencia estadística de que el índice socioeconómico del estudiante aumenta en una unidad, su rendimiento académico se eleva, en promedio y manteniendo constantes las otras variables del modelo, 0,32 puntos en la asignatura. Esta variable no dio evidencia de asociación en el modelo. De acuerdo con los datos, la variable tiene importancia práctica dentro del modelo, con lo cual se comprueba la hipótesis formulada y se confirma que existe relación entre la expectativa de logro que tiene la familia del educando y su rendimiento académico. De igual manera, cuando la expectativa de logro de la familia aumenta en una unidad, controlando las otras variables del modelo, los estudiantes obtienen en promedio 0,17 puntos más en el rendimiento de la asignatura. Así mismo, existe relación entre el rendimiento académico del estudiante y la motivación recibida por este en su familia, pues, cuando el estudiante recibe motivación por parte del grupo familiar, su nota aumenta, en promedio, 0,18 puntos más en la asignatura, manteniendo constantes las otras variables del modelo.

En concordancia con la hipótesis planteada, de acuerdo con los datos obtenidos, al aumentar en una unidad el índice de recursos disponibles en el aula, en promedio y manteniendo constantes las otras variables del modelo, los estudiantes obtienen 0,5 puntos más en su rendimiento académico. Existe evidencia estadística de que, al aumentar en una unidad la escala de percepción del estudiante sobre el dominio de la asignatura que tiene el docente, en promedio y controlando las otras variables del modelo, estos aumentan en 0,03 puntos más su rendimiento académico. $\mathrm{Al}$ aumentar en una unidad la percepción del estudiante sobre la exigencia académica del docente, los estudiantes incrementan su rendimiento académico. la variable tamaño de grupo evidencia que, a mayor cantidad de estudiantes, menor rendimiento académico del estudiante. Aunque existe asociación entre variables, se determina analizar tipo de inteligencia y estilo de aprendizaje, implementando estrategia metodológica que permita evidenciar si existe relación significativa entre estos factores.

Analizando entonces el tipo de aprendizaje muestra que los estudiantes $1,4,8,10,13$ y 20 tienen un marcado dominio por el aprendizaje auditivo; en el estudiante 2, predomina el aprendizaje visual, no obstante también es importante para esta persona el aprendizaje kinestésico; los estudiantes 3, 5, 9, 12, 14, 15, $17,18,22$, domina el aprendizaje kinestésico, sin embargo para los estudiantes 3, 5, y 9 también es significativo el aprendizaje visual y para los estudiantes $12,14,15,17,18, \mathrm{y}$ 22 también es significativo el aprendizaje auditivo; para los estudiantes $6,7,19$, y 23 predomina el aprendizaje auditivo, pero a su vez el aprendizaje kinestésico también es importante; el estudiante 16,tiene aprendizaje auditivo, pero así mismo es significativo el aprendizaje visual y el kinestésico no es nada representativo; los estudiantes 11 y 21 tienen aprendizaje "integral" (término utilizado por el autor del trabajo), dado que, en los tres tipos de aprendizaje obtuvieron un empate con cinco respuestas cada uno. Es importante anotar que el estudiante 15 obtuvo cero respuestas para el aprendizaje visual.

Para esta escala porcentual se tuvo en cuenta el modo de aprendizaje con el mayor número de respuestas en el test, sin entrar a considerar las demás puntuaciones. Es de apreciar que solo un estudiante posee aprendizaje visual y 
dos estudiantes de los 23 tienen las tres formas desarrolladas por igual. Prima el aprendizaje auditivo, seguido del aprendizaje kinestésico.

Se puede concluir con un $95 \%$ de confiabilidad que el grupo de estudiantes tendrá dificultades con el aprendizaje visual, es decir, aprender observando no da buenos resultados, lo que dificultaría el aprendizaje de la estadística, dado que, esta disciplina maneja un alto lenguaje visual a través de gráficas y tablas. Preocupante el caso del estudiante 15, quien según el test el aprendizaje visual no evidencia este modo de aprendizaje, lo que le traería serios problemas en asignaturas como estadística, la cual requiere de la interpretación de resultados de forma visual en tablas y gráficas, lo cual evidencia la relación entre este factor y el rendimiento académico y la búsqueda de otra estrategia dado que el docente enfatizaba en aspectos gráficos.

Para continuar con la investigación, iniciado en el anterior estudio de caso, donde se midió el modo de aprendizaje o forma de aprender, en esta ocasión se aplicó un test de inteligencias múltiples, el cual consistía en 35 preguntas a las cuales se debía contestar con falso o verdadero, de tal manera que se podía identificar qué tipo de inteligencias son las más significativas en cada uno de los estudiantes. A continuación se presenta la tabla de resultados donde se muestran los datos con el tipo de inteligencia dominante o preponderante en cada estudiante. En la tabla 1 . Se omiten todos los valores menores a cuatro respuestas. Como se puede observar, el estudiante 11 se caracteriza por no tener de manera relevante o significativa una inteligencia específica, es decir, no posee alguna inteligencia que lo identifique. Por otra parte, el estudiante 8 demuestra poseer inteligencia lógico - matemática, pero en el mismo, no se destaca ningún otro tipo de inteligencia, por supuesto esto no quiere decir que no las posea, lo que sucede es que no son relevantes. Los estudiantes $4,7,12,14,18$, 23 se identifican con dos tipos de inteligencia. Los estudiantes 1, 2, 3, 10, 16, 17, 19, 20, 21 se destacan con tres tipos de inteligencia, coinciden en algunas. Los estudiantes 6,9 poseen de manera significativa cuatro tipos de inteligencia de manera relevante, así mismo los estudiantes 5,15 y 22 con cinco tipos de inteligencia y el estudiante 13 quien demuestra seis tipos de inteligencia de manera relevante.

Tabla 1. Data de las inteligencias más relevantes en cada uno de 23 estudiantes de la ESAP

\begin{tabular}{|c|c|c|c|c|c|c|c|c|}
\hline \multirow{2}{*}{ Estudiante } & \multicolumn{9}{|c|}{ INTELIGENCIA } \\
\cline { 2 - 10 } & Verb/Ling & Lógi/Mate & Visu/Espac & Kine/Corp & Mus/Ritm & Intraperso & Interperso & Total \\
\hline 1 & 4 & 5 & 0 & 0 & 0 & 0 & 4 & 3 \\
\hline 2 & 4 & 0 & 0 & 0 & 0 & 5 & 5 & 3 \\
\hline 3 & 0 & 0 & 0 & 0 & 5 & 5 & 4 & 3 \\
\hline 4 & 4 & 0 & 4 & 0 & 0 & 0 & 0 & 2 \\
\hline 5 & 0 & 4 & 4 & 4 & 0 & 5 & 4 & 5 \\
\hline 6 & 5 & 0 & 0 & 0 & 4 & 5 & 5 & 4 \\
\hline 7 & 0 & 0 & 0 & 0 & 0 & 5 & 5 & 2 \\
\hline 8 & 0 & 5 & 0 & 0 & 0 & 0 & 0 & 1 \\
\hline 9 & 4 & 0 & 4 & 0 & 0 & 4 & 5 & 4 \\
\hline 10 & 0 & 0 & 0 & 4 & 0 & 4 & 5 & 3 \\
\hline 11 & 0 & 0 & 0 & 0 & 0 & 0 & 0 & 0 \\
\hline 12 & 4 & 0 & 0 & 0 & 4 & 0 & 0 & 2 \\
\hline 13 & 4 & 4 & 0 & 4 & 4 & 4 & 4 & 6 \\
\hline 14 & 0 & 0 & 0 & 0 & 5 & 4 & 0 & 2 \\
\hline 15 & 4 & 0 & 0 & 5 & 4 & 5 & 5 & 5 \\
\hline 16 & 4 & 0 & 0 & 0 & 0 & 5 & 5 & 3 \\
\hline 17 & 0 & 0 & 0 & 0 & 4 & 4 & 5 & 3 \\
\hline 18 & 0 & 0 & 4 & 0 & 0 & 4 & 0 & 2 \\
\hline
\end{tabular}


Eco.Mat

No. 1

Enero-Diciembre 2014 ISSN 1794-8231

PP: 74-86
Inteligencias múltiples y estilos de aprendizaje, su relación con el rendimiento académico de estudiantes en estadística

\begin{tabular}{|c|c|c|c|c|c|c|c|c|}
\hline 19 & 4 & 0 & 0 & 0 & 0 & 5 & 4 & 3 \\
\hline 20 & 5 & 0 & 0 & 0 & 0 & 5 & 4 & 3 \\
\hline 21 & 0 & 4 & 0 & 0 & 0 & 4 & 4 & 3 \\
\hline 22 & 5 & 5 & 4 & 0 & 0 & 5 & 5 & 5 \\
\hline 23 & 0 & 0 & 0 & 0 & 4 & 4 & 0 & 2 \\
\hline Total & 12 & 6 & 5 & 4 & 8 & 18 & 16 & \\
\hline
\end{tabular}

Fuente: Resultados de la aplicación del test a 23 estudiantes de un curso de estadística descriptiva de la Escuela Superior de Administración Pública (ESAP) - Cúcuta.

Se puede concluir que el grupo es bastante heterogéneo, lo que facilitaría el trabajo en equipo, que por sus características produciría buenos resultados porque de hecho se complementarían estas inteligencias para producir excelentes resultados académicos y de formación profesional. asignaturas como matemáticas, estadística, macro y microeconomía, importantes para la formación del administrador público. Así mismo, la inteligencia interpersonal está presente en un porcentaje importante de estudiantes $(69.6 \%)$, situación que se le

Tabla 2. Porcentaje de estudiantes por cada tipo de aprendizaje

\begin{tabular}{|c|c|c|c|c|c|c|}
\hline \multicolumn{6}{|c|}{ PORCENTAJE DE ESTUDIANTES POR CADA TIPO DE INTELIGENCIA } \\
\hline Verb/Ling & Lógi/Mate & Visu/Espac & Kine/Corp & Mus/Ritm & Intrapersonal & Interpersonal \\
\hline 52,2 & 26,1 & 21,7 & 17,4 & 34,8 & 78,3 & 69,6 \\
\hline
\end{tabular}

Fuente: Resultados de la aplicación del test a 23 estudiantes de un curso de estadística descriptiva de la Escuela Superior de Administración Pública (ESAP) - Cúcuta

Las inteligencias intrapersonal e interpersonal son las más significativas con 78.3 y $69.6 \%$ respectivamente. Le siguen en su orden la inteligencia verbal/lingüística, musical/ rítmica, lógica/matemática, visual/espacial y por último la kinestésica/corporal. Es recomendable realizar ejercicios para mejorar el aprendizaje visual ya que esta debilidad no favorece el aprendizaje de encuentra sentido por las características de los estudiantes como personas adultas que trabajan en el sector público y privado o en sus propias microempresas y que según la teoría de Gardner es propia de este tipo de personas, de hecho, tal situación favorece el trabajo en equipo para mejorar el aprendizaje, porque son personas que se relacionan muy bien.

Tabla 3. Cantidad y porcentaje de estudiantes por número de inteligencias relevantes.

\begin{tabular}{|c|c|c|}
\hline $\begin{array}{c}\text { Número de Inteligencias más } \\
\text { Significativas }\end{array}$ & $\begin{array}{c}\text { Número de } \\
\text { Estudiantes }\end{array}$ & $\begin{array}{c}\text { Porcentaje de } \\
\text { Estudiantes }\end{array}$ \\
\hline 0 & 1 & 4,3 \\
\hline 1 & 1 & 4,3 \\
\hline 2 & 6 & 26,1 \\
\hline 3 & 9 & 39,1 \\
\hline 4 & 2 & 8,7 \\
\hline 5 & 3 & 13,0 \\
\hline 6 & 1 & 4,3 \\
\hline Total & 23 & 100,0 \\
\hline
\end{tabular}

Fuente: Resultados de la aplicación del test a 23 estudiantes de un curso de estadística descriptiva de la Escuela Superior de Administración Pública (ESAP) - Cúcuta. 
José Joaquín Martínez-Lozano, Mawency Vergel-Ortega, Henry de Jesús Gallardo-Pérez

Tabla No. 4. Frecuencia Número de tipos de inteligencia por estudiante Inteligencia (frecuencias)

\begin{tabular}{|c|c|c|c|c|}
\hline & \multicolumn{2}{|c|}{ Respuestas } & \multirow{2}{*}{$\begin{array}{l}\text { Porcentaje de } \\
\text { casos }\end{array}$} \\
\hline & & $\mathrm{N}$ & Porcentaje & \\
\hline \multirow[t]{8}{*}{ Tipo de Inteligencia $^{a}$} & Inteligencia Verb/Ling & 12 & 17,4 & 54,5 \\
\hline & Inteligencia Lógi/Mate & 6 & 8,7 & 27,3 \\
\hline & Visu/Espac & 5 & 7,2 & 22,7 \\
\hline & Kine/Corp & 4 & 5,8 & 18,2 \\
\hline & Mus/Ritm & 8 & 11,6 & 36,4 \\
\hline & Intrapersonal & 18 & 26,1 & 81,8 \\
\hline & Interpersonal & 16 & 23,2 & 72,7 \\
\hline & Total & 69 & 100,0 & 313,6 \\
\hline
\end{tabular}

a. Grupo de dicotomía tabulado en el valor 1 .

Los estudiantes de Administración de Empresas de la ESAP tienen alrededor de 3,13 tipos de inteligencia a la vez, como se observa en tabla 4.

Tabla No. 5 Tipo de inteligencia vs nivel de aprendizaje

\begin{tabular}{|c|c|c|c|c|c|c|}
\hline & & & \multicolumn{3}{|c|}{ Nivel de Aprendizaje ${ }^{b}$} & \multirow[b]{2}{*}{ Total } \\
\hline & & & Bajo & Medio & Alto & \\
\hline \multirow{14}{*}{$\begin{array}{l}\text { Tipo de } \\
\text { Inteligencia }\end{array}$} & Inteligencia & Recuento & 3 & 16 & 29 & 12 \\
\hline & Verb/Ling & $\%$ dentro de \$Inteligencia & $25,0 \%$ & $133,3 \%$ & $241,7 \%$ & \\
\hline & Inteligencia & Recuento & 2 & 7 & 15 & 6 \\
\hline & Lógi/Mate & $\%$ dentro de \$Inteligencia & $33,3 \%$ & $116,7 \%$ & $250,0 \%$ & \\
\hline & Visu/Espac & Recuento & 1 & 6 & 13 & 5 \\
\hline & & $\%$ dentro de \$Inteligencia & $20,0 \%$ & $120,0 \%$ & $260,0 \%$ & \\
\hline & Kine/Corp & Recuento & 1 & 5 & 10 & 4 \\
\hline & & $\%$ dentro de \$Inteligencia & $25,0 \%$ & $125,0 \%$ & $250,0 \%$ & \\
\hline & Mus/Ritm & Recuento & 2 & 12 & 18 & 8 \\
\hline & & $\%$ dentro de \$Inteligencia & $25,0 \%$ & $150,0 \%$ & $225,0 \%$ & \\
\hline & Intrapersonal & Recuento & 5 & 27 & 40 & 18 \\
\hline & & $\%$ dentro de \$Inteligencia & $27,8 \%$ & $150,0 \%$ & $222,2 \%$ & \\
\hline & Interpersonal & Recuento & 5 & 22 & 37 & 16 \\
\hline & & $\%$ dentro de \$Inteligencia & $31,3 \%$ & $137,5 \%$ & $231,3 \%$ & \\
\hline \multicolumn{2}{|l|}{ Total } & Recuento & 6 & 34 & 48 & 22 \\
\hline
\end{tabular}

Los porcentajes y los totales se basan en los encuestados.

a. Grupo de dicotomía tabulado en el valor 1.

b. Grupo 
Eco.Mat

No. 1

Enero-Diciembre 2014 ISSN 1794-8231

PP: $74-86$
Tienen un desempeño alto en el aprendizaje de Estadística aproximadamente 2,4 estudiantes que muestran inteligencia verbal, 2,5 con inteligencia lógico matemática, 2,6 con inteligencia visual especial, 2,5 kinésico corporal, 2,2 musical, 2,3 interpersonal y 2,2 intrapersonal.

Tabla No. 6. Tipo de inteligencia-rendimiento académico

\begin{tabular}{|c|c|c|c|c|c|c|}
\hline & & & & ndimien & & \\
\hline & & & 2,00 & 3,00 & 4,00 & Total \\
\hline Tipo de & Inteligencia & Recuento & 2 & 8 & 2 & 12 \\
\hline Inteligencia ${ }^{\mathrm{a}}$ & Verb/Ling & $\begin{array}{l}\% \text { dentro de } \\
\text { \$Inteligencia }\end{array}$ & $16,7 \%$ & $66,7 \%$ & $16,7 \%$ & \\
\hline & Inteligencia & Recuento & 0 & 2 & 4 & 6 \\
\hline & Lógi/Mate & $\begin{array}{l}\% \text { dentro de } \\
\text { \$Inteligencia }\end{array}$ & $0,0 \%$ & $33,3 \%$ & $66,7 \%$ & \\
\hline & Visu/Espac & Recuento & 0 & 2 & 3 & 5 \\
\hline & & $\begin{array}{l}\% \text { dentro de } \\
\text { \$Inteligencia }\end{array}$ & $0,0 \%$ & $40,0 \%$ & $60,0 \%$ & \\
\hline & Kine/Corp & Recuento & 0 & 3 & 1 & 4 \\
\hline & & $\begin{array}{l}\% \text { dentro de } \\
\text { \$Inteligencia }\end{array}$ & $0,0 \%$ & $75,0 \%$ & $25,0 \%$ & \\
\hline & Mus/Ritm & Recuento & 4 & 3 & 1 & 8 \\
\hline & & $\begin{array}{l}\% \text { dentro de } \\
\text { \$Inteligencia }\end{array}$ & $50,0 \%$ & $37,5 \%$ & $12,5 \%$ & \\
\hline & Intrapersonal & Recuento & 4 & 9 & 5 & 18 \\
\hline & & $\begin{array}{l}\% \text { dentro de } \\
\text { \$Inteligencia }\end{array}$ & $22,2 \%$ & $50,0 \%$ & $27,8 \%$ & \\
\hline & Interpersonal & Recuento & 2 & 9 & 5 & 16 \\
\hline & & $\begin{array}{l}\% \text { dentro de } \\
\text { \$Inteligencia }\end{array}$ & $12,5 \%$ & $56,3 \%$ & $31,3 \%$ & \\
\hline
\end{tabular}

Los porcentajes y los totales se basan en los encuestados.

a. Grupo de dicotomía tabulado en el valor 1.

En general, estudiantes con inteligencia interpersonal e intrapersonal tienen rendimiento medio, inteligencia lógico- matemático tienen rendimiento superior, inteligencia kine-corporal, interpersonal e intrapersonal tienen rendimiento medio. 
José Joaquín Martínez-Lozano, Mawency Vergel-Ortega, Henry de Jesús Gallardo-Pérez

Gráfica 2. Tipo de inteligencia- estilo de aprendizaje
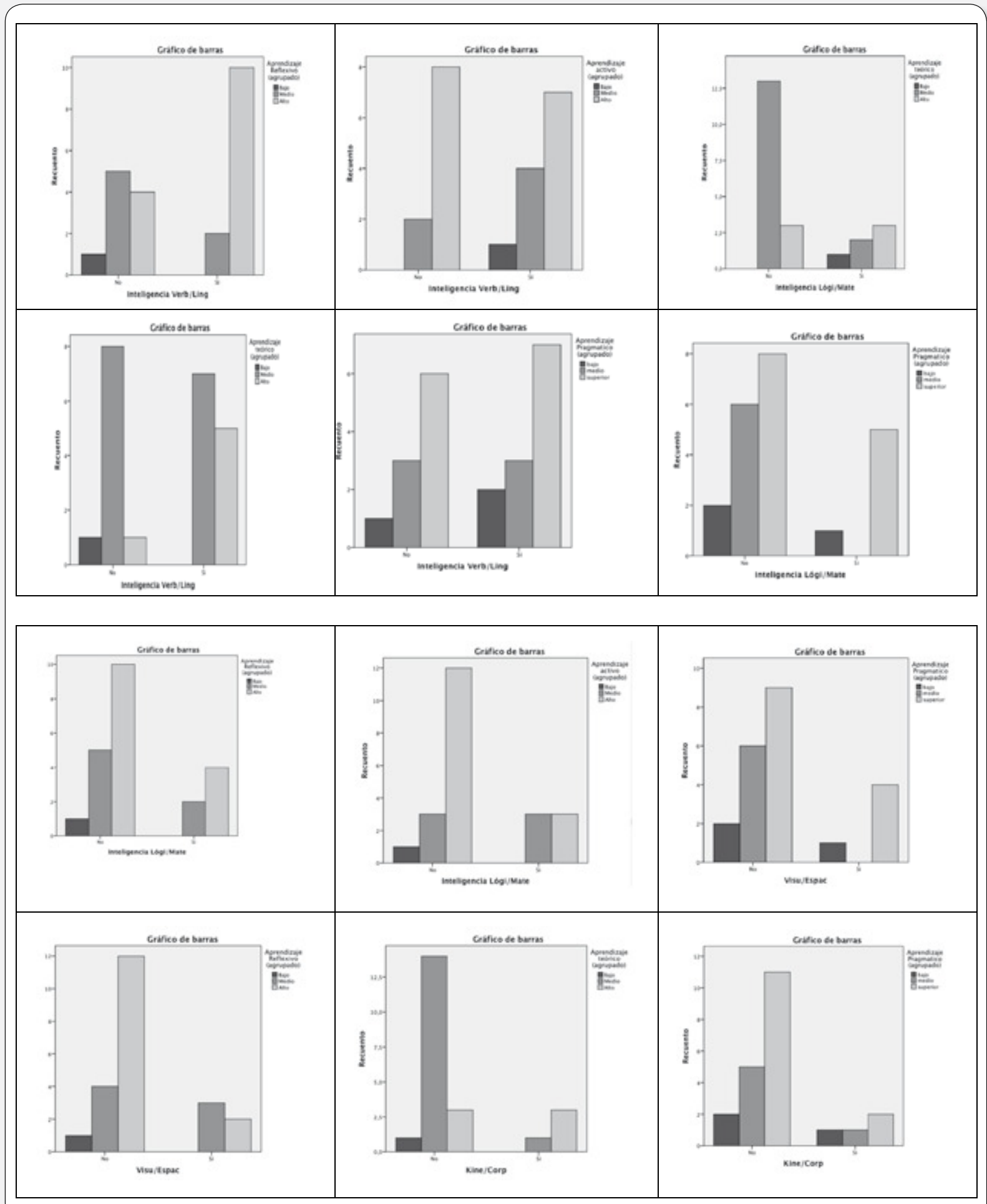

Eco.Mat

Cúcuta-Colombia

Vol. 5

No. 1

Enero-Diciembre 2014

ISSN $1794-8231$

PP: $74-86$

83 


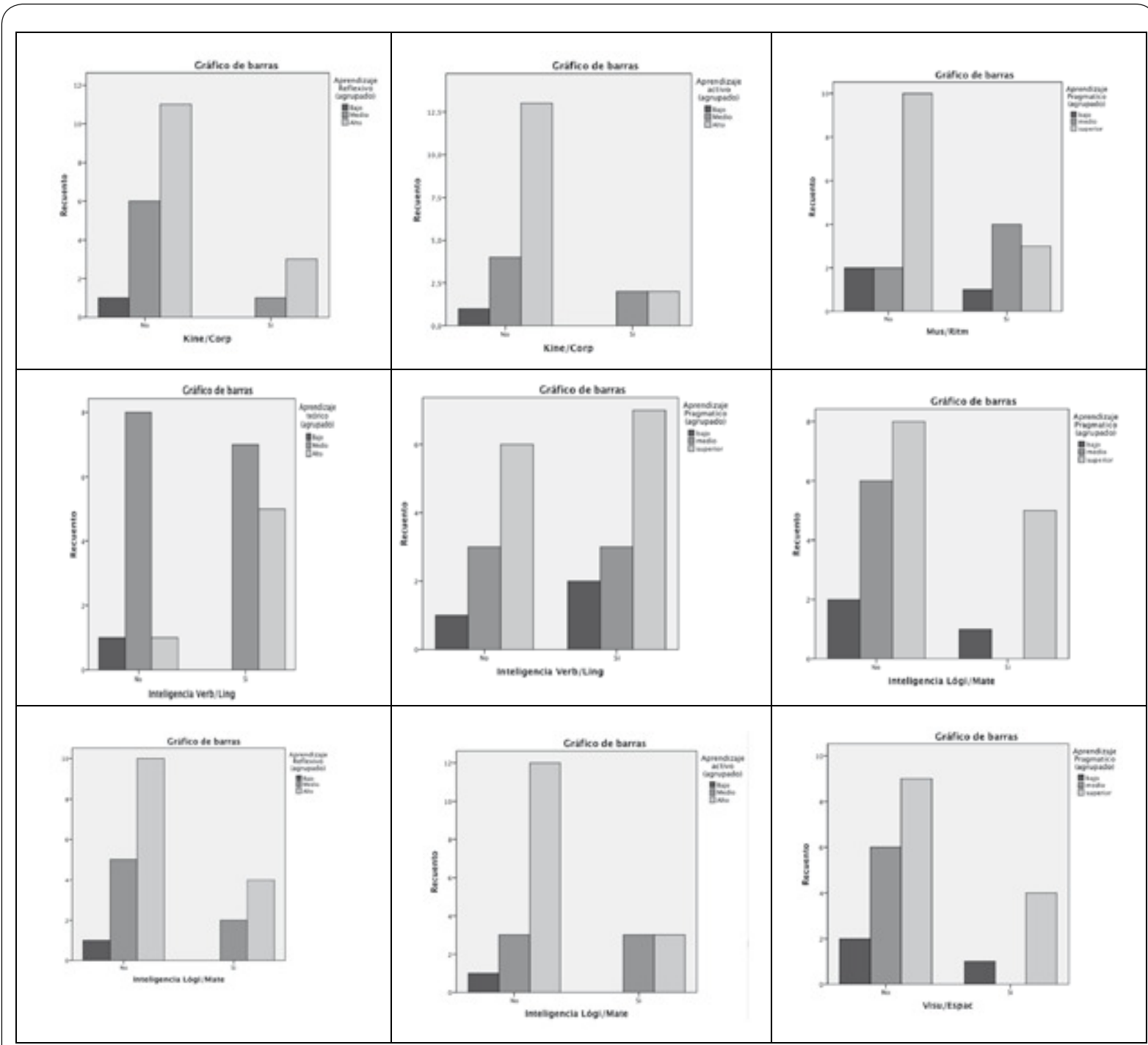

No existe relación entre aprendizaje teórico, aprendizaje pragmático y tipo de inteligencia verbal la variable inteligencia verbal solo explica en $0,4 \%$ el aprendizaje teórico, en $2 \%$ el aprendizaje reflexivo

La inteligencia lógico-matemático explica el aprendizaje teórico en 23,8\%; el aprendizaje reflexivo en $5 \%$, aprendizaje activo en $2 \%$. La inteligencia visual espacial explica en $40 \%$ el aprendizaje teórico, aprendizaje pragmático en $20 \%$ relación negativa, aprendizaje activo $13 \%$ relación negativa, aprendizaje reflexivo en $12 \%$, la inteligencia kinestésico-corporal explica en $50 \%$ el aprendizaje teórico, aprendizaje pragmático en $11 \%$ relación negativa, aprendizaje activo lo explica en $16 \%$. Inteligencia musical explica el aprendizaje pragmático en $26 \%$, aprendizaje reflexivo $0,06 \%$, aprendizaje activo en $46 \%$, aprendizaje teórico en $41 \%$. Inteligencia intrapersonal explica en $16 \%$ (relación negativa) a 1 aprendizaje pragmático, 35,2\% aprendizaje reflexivo, $40 \%$ al aprendizaje activo, 45,4\% aprendizaje teórico, $\mathrm{La}$ inteligencia interpersonal explica en $0,2 \%$ aprendizaje pragmático, 55 al aprendizaje reflexivo, no existe relación alguna con el aprendizaje activo. 


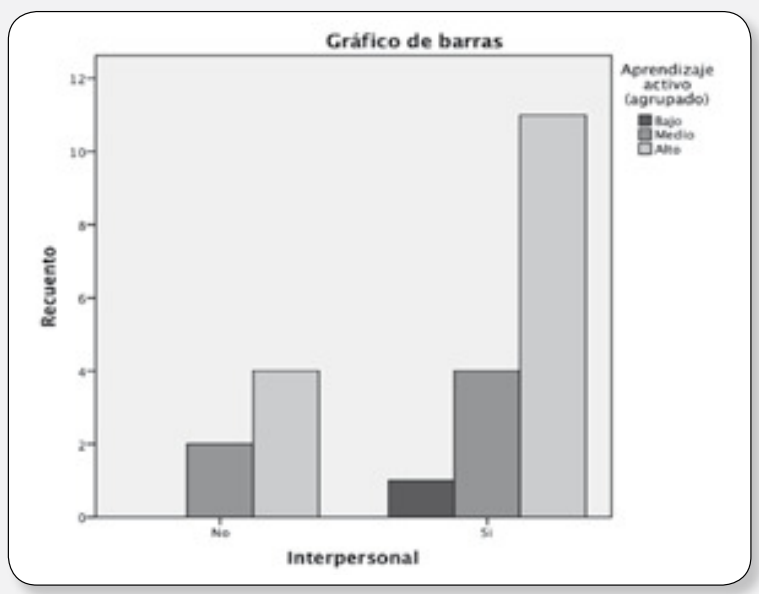

Se puede apreciar entre dos y tres las cantidades de inteligencias más relevantes que poseen los estudiantes objeto de estudio con $65.2 \%$; tal situación indica que el grupo puede dar buen rendimiento sabiendo explotar sus fortalezas a través del modelo pedagógico y las estrategias didácticas implementadas.

\section{CONCLUSIONES}

Estilo de aprendizaje, género, expectativa de logro de la familia, motivación de la familia, así como exigencia del docente son factores asociados al rendimiento académico del estudiante de la Escuela superior de Administración Pública en el aprendizaje de la estadística. El tipo de inteligencia está asociado e influye en el comportamiento del grupo y potencia habilidades de cada estudiante al utilizar metodologías que integren los estudiantes en torno a roles según aptitudes, estilo de aprendizaje y tipo de inteligencia, logrando con esto el desarrollo de proyectos aplicados en estadística.

Considerar "inteligencias", "capacidades" o "fortalezas" a las facultades más o menos desarrolladas en las personas, a los docentes les resulta de suma utilidad analizarlas en los estudiantes, ya que permite comprenderlos más y delinear actividades y estrategias metodológicas apropiadas para obtener los máximos resultados en su rendimiento académico.
Las inteligencias intrapersonal e interpersonal son las más significativas con 78.3 y $69.6 \%$ respectivamente. Le siguen en su orden la inteligencia verbal/lingüística, musical/ rítmica, lógica/matemática, visual/espacial y por último la kinestésica/corporal. Una de las debilidades de los grupos es la inteligencia visual/espacial. Es recomendable realizar ejercicios para mejorar ya que esta debilidad no favorece el desarrollo de competencias de asignaturas como matemáticas, estadística, macro y microeconomía, importantes para la formación del administrador público.

La inteligencia interpersonal está presente en un porcentaje importante de estudiantes $(69.6 \%)$, situación que se le encuentra sentido por las características de los estudiantes como personas adultas que trabajan en el sector público y privado o en sus propias microempresas y que según la teoría de Gardner es propia de este tipo de personas, de hecho, tal situación favorece el trabajo en equipo para mejorar el aprendizaje, porque son personas que se relacionan muy bien

Tienen un desempeño alto en el aprendizaje de Estadística aproximadamente 2,4 estudiantes que muestran inteligencia verbal, 2,5 con inteligencia lógico matemática, 2,6 con inteligencia visual especial, 2,5 kinésico corporal, 2,2 musical, 2,3 interpersonal y 2,2 intrapersonal.

En general, estudiantes con inteligencia interpersonal e intrapersonal tienen un rendimiento medio, inteligencia lógicomatemático tienen un rendimiento superior, inteligencia kine-corporal, interpersonal e intrapersonal tienen rendimiento medio.

No se encontró relación significativa entre el tipo de inteligencia y el tipo de aprendizaje de los estudiantes de administración de empresas.
Enero-Diciembre 2014 ISSN 1794-8231 PP: 74-86 
Eco.Mat

No. 1

Enero-Diciembre 2014 ISSN 1794-8231

PP: $74-86$

ARMSTRONG, Thomas. Las inteligencias múltiples en el aula. Manantial.1999

BORSANI, María José. Adecuaciones curriculares. Novedades Educativas. 2001

CRESPO ALLENDE, Nina. Inteligencias múltiples y Aprendizajes Escolares. http:// rehue.csociales.uchile.cl/rehuehome/ facultad/publicaciones/Talon/talon5/

CRESPO ALLENDE, Nina. Tesis Doctoral en Lingüística Aplicada. Universidad de Chile, Facultad de Ciencias Sociales

GARDNER, Howard. Estructuras de la mente. Fondo de Cultura económica. México.1994.

GARBANZO, G (2007). Factores asociados al rendimiento académico en estudiantes universitarios, una reflexión desde la calidad de la educación superior pública. En: Revista Educación 31(1), p.44

INEE (2008). PISA en el aula: lectura. México.

KRUSCHE, H. (2006). La rana sobre la mantequilla: Fundamentos de la Programación Neurolingüística. Editorial Sirio, S.A. Málaga -España.

LAPALMA, Fernando. Inteligencias Múltiples.www.galeon.hispavista.com/ aprenderaaprender/intmultiples/lapalma.htm

LLECE (2001). Primer estudio internacional comparativo sobre lenguaje, matemática y factores asociados para alumnos de tercer y cuarto grado de la educación básica. Informe técnico. Unesco, Santiago, Chile.

LÓPEZ PÉREZ, Ricardo. En torno a Inteligencias Múltiples. Revista Enfoques Educacionales Vol. I No 2 1998. Departamento de Educación, Facultad de Ciencias Sociales. Universidad de Chile.

LUPIAÑEZ, Miguel. Inteligencias Múltiples y desarrollo personal. Raczynski, D. y Muñoz, G. (2005). Efectividad escolar y cambio educativo en condiciones de pobreza en Chile. Gobierno de Chile, Ministerio de Educación, Santiago: Maval Ltda.

ROMÁN, M \& MURILLO, F (2011). América Latina: violencia entre estudiantes y desempeño escolar. En: Revista CEPAL 104 (agosto, 2011) Consultado el 9 de Abril de 2012. 